\title{
Shear-rate dependence of thermodynamic properties of the Lennard-Jones truncated and shifted fluid by molecular dynamics simulations
}

Cite as: Phys. Fluids 31, 063103 (2019); https://doi.org/10.1063/1.5090489

Submitted: 28 January 2019 . Accepted: 22 May 2019. Published Online: 19 June 2019

Martin P. Lautenschlaeger (D), and Hans Hasse

\section{ARTICLES YOU MAY BE INTERESTED IN}

Analytical study of oscillatory flow of Maxwell fluid through a rectangular tube

Physics of Fluids 31, 063102 (2019); https://doi.org/10.1063/1.5100220

Deformation characteristics and energy conversion during droplet impact on a water surface

Physics of Fluids 31, 062108 (2019); https://doi.org/10.1063/1.5099228

Three-dimensional numerical simulation of bubble rising in viscous liquids: A conservative phase-field lattice-Boltzmann study

Physics of Fluids 31, 063106 (2019); https://doi.org/10.1063/1.5096390

\section{Scilight Highlights of the best new research Scilight in the physical sciences}




\title{
Shear-rate dependence of thermodynamic properties of the Lennard-Jones truncated and shifted fluid by molecular dynamics simulations
}

\author{
Cite as: Phys. Fluids 31, 063103 (2019); doi: 10.1063/1.5090489 \\ Submitted: 28 January 2019 - Accepted: 22 May 2019 • \\ Published Online: 19 June 2019

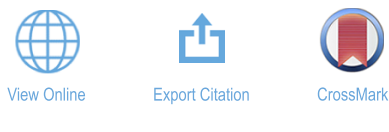

Martin P. Lautenschlaeger ${ }^{\mathrm{a})}$ (D) and Hans Hasse

\begin{abstract}
AFFILIATIONS
Laboratory of Engineering Thermodynamics (LTD), University of Kaiserslautern, Erwin-Schrödinger-Straße 44, 67663 Kaiserslautern, Germany
\end{abstract}

a) Electronic mail: Martin.Lautenschlaeger@mv.uni-kl.de

\begin{abstract}
It was shown recently that using the two-gradient method, thermal, caloric, and transport properties of fluids under quasi-equilibrium conditions can be determined simultaneously from nonequilibrium molecular dynamics simulations. It is shown here that the influence of shear stresses on these properties can also be studied using the same method. The studied fluid is described by the Lennard-Jones truncated and shifted potential with the cut-off radius $r_{\mathrm{c}}^{*}=2.5 \sigma$. For a given temperature $T$ and density $\rho$, the influence of the shear rate on the following fluid properties is determined: pressure $p$, internal energy $u$, enthalpy $h$, isobaric heat capacity $c_{\mathrm{p}}$, thermal expansion coefficient $\alpha_{\mathrm{p}}$, shear viscosity $\eta$, and self-diffusion coefficient $D$. Data for 27 state points in the range of $T \in[0.7,8.0]$ and $\rho \in[0.3,1.0]$ are reported for five different shear rates $(\dot{\gamma} \in[0.1,1.0])$. Correlations for all properties are provided and compared with literature data. An influence of the shear stress on the fluid properties was found only for states with low temperature and high density. The shear-rate dependence is caused by changes in the local structure of the fluid which were also investigated in the present work. A criterion for identifying the regions in which a given shear stress has an influence on the fluid properties was developed. It is based on information on the local structure of the fluid. For the self-diffusivity, shear-induced anisotropic effects were observed and are discussed.
\end{abstract}

Published under license by AIP Publishing. https://doi.org/10.1063/1.5090489

\section{INTRODUCTION}

Experimental studies show that the viscosity of complex fluids such as colloidal solutions, suspensions, or polymer melts may decrease when shear is applied. Therefore, this effect is called shear thinning. However, there is almost no experimental data on the shear-rate dependence of other fluid properties than the viscosity. Also for the viscosity, experimental data on shear thinning of simple fluids like argon are scarce. The reason for this is that for simple fluids, very high shear rates, as they appear in cavitation phenomena or detonation scenarios, have to be applied to observe this effect. This is difficult to realize in experimental setups that are suited for measuring fluid properties. An alternative to experimental studies is computer simulations. Molecular dynamics (MD) simulations are particularly suited for studying the effect of the shear rate on fluid properties. Such simulations are used here for studying the shear-rate dependence of properties of a simple, monatomic, and dispersive model fluid. Understanding the shear-rate dependence of such simple fluids is a prerequisite for understanding this effect in more complex fluids and also for developing theories for describing it. ${ }^{1,2}$

Simple fluids are often modeled using potentials of the Lennard-Jones (LJ) type. The whole class of LJ-type potentials is subdivided into the full LJ potential, the computationally particularly efficient truncated LJ potential, as well as the truncated and shifted LJ potential. For the truncation, different cut-off radii $r_{\mathrm{c}}$ have been used in the literature. For most of these potential models, data on equilibrium thermodynamic properties, transport properties, interfacial properties, and the phase behavior have been studied extensively. ${ }^{3-}$ However, there is not much literature data on the shear-rate 
dependence of properties of LJ-type fluids. In most of the early studies, shear-rate effects were determined close to the triple point of either the LJ fluid ${ }^{10,11}$ or the truncated LJ fluid with the cut-off radius $r_{\mathrm{c}}^{*}=2.5 \sigma$ (LJT fluid), ${ }^{12-15}$ where $\sigma$ is the size parameter of the LJ potential. Only a few studies report data for a wide range of thermodynamic states and for the LJT fluid only. There, the shear-rate dependence of the pressure, internal energy, and shear viscosity ${ }^{16-18}$ as well as the self-diffusion coefficient ${ }^{17,18}$ was investigated. But very little is known on the shear-rate dependence of other properties. Anisotropy of the self-diffusivity of LJ-type fluids has been observed by some authors, ${ }^{14,15,19}$ but a systematic study is lacking. Structural changes of the LJ-type fluid under shear have been studied for some examples by other authors ${ }^{14,20-26}$ and were used to explain the shearrate dependence of the macroscopic fluid properties. To the best of our knowledge, there are no reports on the shear-rate dependence of fluid properties of other simple fluids than those of the LJ-types discussed in this paragraph.

Therefore, in the present study, the shear-rate dependence of different thermal, caloric, and transport properties of the LJ fluid truncated and shifted at the cut-off radius $r_{\mathrm{c}}^{*}=2.5 \sigma$ (LJTS fluid) was studied systematically using the recently developed two-gradient method. ${ }^{9,27}$ This nonequilibrium molecular dynamics (NEMD) simulation method is characterized by the superposition of a velocity gradient $\partial \boldsymbol{v}_{x} / \partial y$ and a temperature gradient $\partial T / \partial y$. The velocity gradient ensures that the shear-rate dependence of the different fluid properties can be studied. The following properties were determined in the present study: pressure $p$, internal energy $u$, enthalpy $h$, isobaric heat capacity $c_{\mathrm{p}}$, thermal expansion coefficient $\alpha_{\mathrm{p}}$, shear viscosity $\eta$, and self-diffusion coefficient $D$. The study of $c_{\mathrm{p}}$ and $\alpha_{\mathrm{p}}$ is enabled by the temperature gradient. In addition, also data for the thermal conductivity $\lambda$ are given in the supplementary material. These, however, are subject to large statistical uncertainties that are due to numerical artifacts, which are further described below. The temperature gradient was small enough to ensure that it does not significantly influence the results of the fluid properties. ${ }^{8,9,27}$ The general applicability of the two-gradient method for studying the shear-rate dependence of transport properties has already been shown in a previous study ${ }^{27}$ using some examples. It was also shown in previous works of our group ${ }^{8,9}$ that in simulations with the two-gradient method in contrast to other NEMD methods, no drift of the temperature and the total energy is observed; see discussion in Ref. 28. Moreover, the thermostatting method that is used in the two-gradient method does not require any knowledge of the flow profile. This is known to be particularly important for avoiding structural artifacts when studying a non-Newtonian fluid behavior. ${ }^{20,21,29,30}$ In a single run with the two-gradient method, all aforementioned properties can be determined simultaneously for a given shear rate $\dot{\gamma}=\partial \boldsymbol{v}_{x} / \partial y$, temperature $T$, and density $\rho$. The method can also be used to study structural phenomena. Furthermore, for the self-diffusivity, even anisotropic effects can be studied which is not the case for the other transport properties $(\lambda$ and $\eta)$.

In the present work, 27 thermodynamic points of the LJTS fluid in the liquid and supercritical regime $(T \in[0.7,8.0]$ and $\rho \in[0.3$, $1.0])$ were investigated. For each state point, five different shear rates $\dot{\gamma} \in[0.1,1.0]$ were studied. Literature data for a comparison are only available for $\eta$ and $D .{ }^{16-18}$ Where comparisons are possible, the present results agree with those from the literature.
The comprehensive data set from the present work was used to investigate which state points are most sensitive to a shear-rate effect, and a criterion to measure this shear-rate sensitivity is introduced. The shear-rate dependence of macroscopic fluid properties is closely related to changes of the local structure of the fluid, which are also studied. Furthermore, the anisotropy of the self-diffusivity of the LJTS fluid under shear is investigated systematically and the results are compared with predictions from the Stokes-Einstein relation. ${ }^{3}$

This paper is organized as follows: in Sec. II, the LJTS force field, the simulation setup, and the method that was used for analyzing the structure of a fluid and its shear-rate sensitivity are specified. In Sec. III, the scope of the study is described. The simulation results of the fluid properties, the results of the structural analysis, and the correlations are presented in Sec. IV. Moreover, in that section, findings are explained based on structural changes of the fluid, the anisotropy of the self-diffusivity is shown, and the applicability of the Stokes-Einstein relation is discussed. Finally, conclusions are drawn in Sec. V.

\section{MOLECULAR MODEL AND SIMULATION DETAILS}

\section{A. Molecular model}

The LJTS potential which was studied in the present work is

$$
u^{\mathrm{LJTS}}\left(r_{i j}\right)=\left\{\begin{array}{ll}
u^{\mathrm{LJ}}\left(r_{i j}\right)-u^{\mathrm{LJ}}\left(r_{\mathrm{c}}\right), & \forall r_{i j}<r_{\mathrm{c}} \\
0, & \forall r_{i j} \geq r_{\mathrm{c}}
\end{array},\right.
$$

where

$$
u^{\mathrm{LJ}}\left(r_{i j}\right)=4 \varepsilon\left[\left(\sigma / r_{i j}\right)^{12}-\left(\sigma / r_{i j}\right)^{6}\right] .
$$

Dispersive attractions are described with the energy parameter $\varepsilon$. The repulsion is described with the size parameter $\sigma$. The distance between two LJTS particles $i$ and $j$ is $r_{i j}$. The cut-off radius is set to $r_{\mathrm{c}}^{*}=2.5 \sigma$ in all simulations of the present work. Each particle has the mass $m=1$ such that the mass density $\rho_{\mathrm{m}}$ and the number density $\rho$ are identical. In the following, all observables are given in reduced LJ units, cf. Table II in Appendix A. Using the conversion rules given in Table II in Appendix A and using the LJTS model parameters for real substances (argon, krypton, xenon, and methane) from Ref. 32, the values of all data from the present work can be converted to the corresponding properties of the real substances.

\section{B. Simulation setup}

The two-gradient method which is used in the present work is described in detail in two recent publications of our group. ${ }^{9,27}$ Therefore, only a brief introduction into the method is given here.

Figure 1 shows the simulation scenario. In the present study, the simulation box is cubic. However, as was discussed in Refs. 9 and 27 , the two-gradient method can be applied for any cuboid system. Initially, the simulation box is homogeneously filled with the LJTS fluid, for which the mean particle density is prescribed. There are no solid boundaries such that there are no surface effects. Periodic boundary conditions are applied in all directions. The edge length $L$ is set such that the system contains about 10000 particles. Finitesize effects ${ }^{33-35}$ are not important here as the lower limit of the edge length is set to $L_{\min }=24$. This ensures that even the self-diffusion 

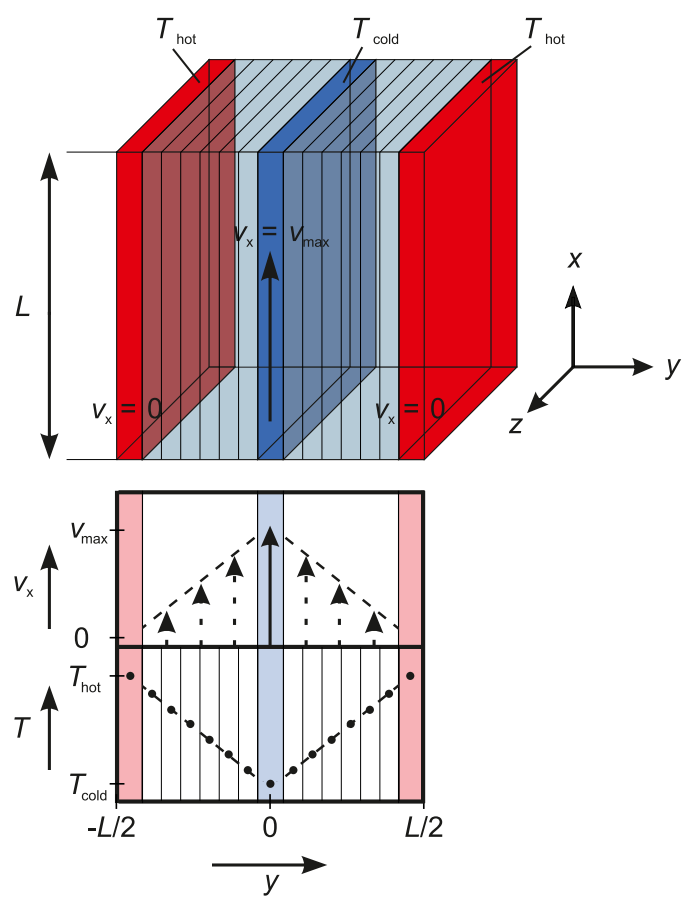

FIG. 1. Scheme of the simulation setup of the two-gradient method as it was applied in the present work. The control volumes in which both the temperature and the velocity are prescribed simultaneously are marked in blue and red. The control volumes in which only the temperature is prescribed are inbetween. The shear flow is in the $+x$-direction. Exemplary temperature and velocity profiles are depicted at the bottom. The dots indicate the mean temperature that is adjusted locally in each control volume. The dashed lines and arrows indicate responses. The bins are only schematic.

coefficient, which is highly sensitive to the system size, ${ }^{36,37}$ is not systematically affected.

A velocity gradient $\partial \boldsymbol{v}_{x} / \partial y=\dot{\gamma} \in[0.1,1.0]$ and a temperature gradient $\partial T / \partial y=0.03$ are applied simultaneously. The velocity is prescribed in control volumes at the two borders of the box in the $y$-direction and in the center of the box only. By contrast, thermostatization by velocity scaling is applied binwise to the entire simulation volume to induce a temperature gradient in the $y$-direction. The width of each velocity control volume is $0.5 \sigma$. The width of each control volume in which the temperature is prescribed is $2 / 3 \sigma$. The thermostatization in such small control volumes ensures that effects of viscous heating do not show up while the temperature gradient is small and perfectly linear even for large values of $\dot{\gamma}$.

With the two-gradient method, the properties $p, u, h, c_{\mathrm{p}}, \alpha_{\mathrm{p}}$, $\lambda, \eta$, and $D$ are determined using classical thermodynamic relations. These can be applied here as the local equilibrium assumption holds, as has been discussed in our previous works ${ }^{9,27}$ and is also shown in the following. The simulation box is uniformly discretized in the $y$-direction using 48 bins with the width $\Delta y=L / 48$ each. Positions and sizes of the bins are not identical with those of the velocity and temperature control volumes. In each bin, the observables density $\rho$, temperature $T$, velocity $\boldsymbol{v}_{x}$, internal energy $u$, enthalpy $h$, heat flux $\boldsymbol{j}_{y}$, stress $\boldsymbol{\sigma}_{x y}$, and self-diffusivity $\boldsymbol{D}$ are recorded during the simulation run via block-averaging. The observables $\boldsymbol{j}_{y}$ and $\boldsymbol{\sigma}_{x y}$ are determined with the Irving and Kirkwood approach. ${ }^{38}$ The $k$-component of $\boldsymbol{D}$ is determined as

$$
\boldsymbol{D}_{k}=\frac{1}{2 N_{\mathrm{bin}} \Delta t}\left[\sum_{i}^{N_{\mathrm{bin}}}\left(\boldsymbol{r}_{i, k}(t+\Delta t)-\boldsymbol{r}_{i, k}(t)-\left\langle\boldsymbol{v}_{k, \mathrm{dir}}\right\rangle \Delta t\right)^{2}\right],
$$

which describes the diffusive movement of a particle $i$ (with the position $\boldsymbol{r}_{i}$ ) along the $k$-direction within the time period $\Delta t$. Here, $N_{\text {bin }}$ is the number of particles per bin. The contribution of the convective shear flow $\boldsymbol{v}_{k \text {,dir }}$ (i.e., the directed macroscopic flow velocity in the $k$ direction) is subtracted. $\boldsymbol{v}_{k \text {,dir }}$ is time-averaged over $\Delta t$ as indicated by the angle brackets.

The profiles of different observables are exemplarily shown in Fig. 2. From the profiles, the properties $p, u, h$, and $D$ are determined directly as mean values. Data from bins in which both the velocity and the temperature are prescribed simultaneously are excluded from the sampling. The data from the other regions are averaged and reported as functions of $\bar{T}, \bar{\rho}$, and $\dot{\gamma}$, where the bar indicates the spatial averages (cf. Fig. 2). The bars over $T$ and $\rho$ are only used in the present section, where also spatial results are shown. In Secs. III-V, they are omitted for simplicity. The properties $\lambda, \eta, c_{\mathrm{p}}$, and $\alpha_{\mathrm{p}}$ are determined via postprocessing as second-order properties. Therefore, least squares regressions (cf. red-dashed lines in Fig. 2) are separately conducted on the left and the right side in the unconstrained regions. Second-order polynomials are fitted to the profiles of the density, the temperature, the velocity, and the enthalpy. Linear polynomials are fitted to the profiles of the heat flux and the shear stress. $\lambda$ and $\eta$ are determined binwise from Fourier's law

$$
\boldsymbol{j}_{y}=-\lambda \frac{\partial T}{\partial y}
$$

and Newton's law

$$
\boldsymbol{\sigma}_{x y}=-\eta \frac{\partial \boldsymbol{v}_{x}}{\partial y}=-\eta \dot{\gamma},
$$

respectively. $c_{\mathrm{p}}$ and $\alpha_{\mathrm{p}}$ are determined binwise as

$$
c_{\mathrm{p}}=\frac{\partial h}{\partial T}=\frac{\partial h}{\partial y}\left(\frac{\partial T}{\partial y}\right)^{-1}
$$

and

$$
\alpha_{\mathrm{p}}=-\frac{\partial(\ln \rho)}{\partial T}=-\frac{\partial(\ln \rho)}{\partial y}\left(\frac{\partial T}{\partial y}\right)^{-1} .
$$

All gradients in Eqs. (4)-(7) are approximated binwise by applying the finite difference method to the regression fits.

The time step is $\Delta \tau=0.002$. A simulation run consists of initially 500000 time steps in which a steady state with a stable temperature and velocity gradient is established. It is followed by a production run of 2000000 time steps. During the production run, a block-averaging with a block size of 10000 time steps was applied and the standard deviations of the block data for all observables were determined. These standard deviations are considered here as the statistical uncertainties of the corresponding variables. The statistical uncertainties of the aforementioned second-order properties are estimated following Ref. 39 as 

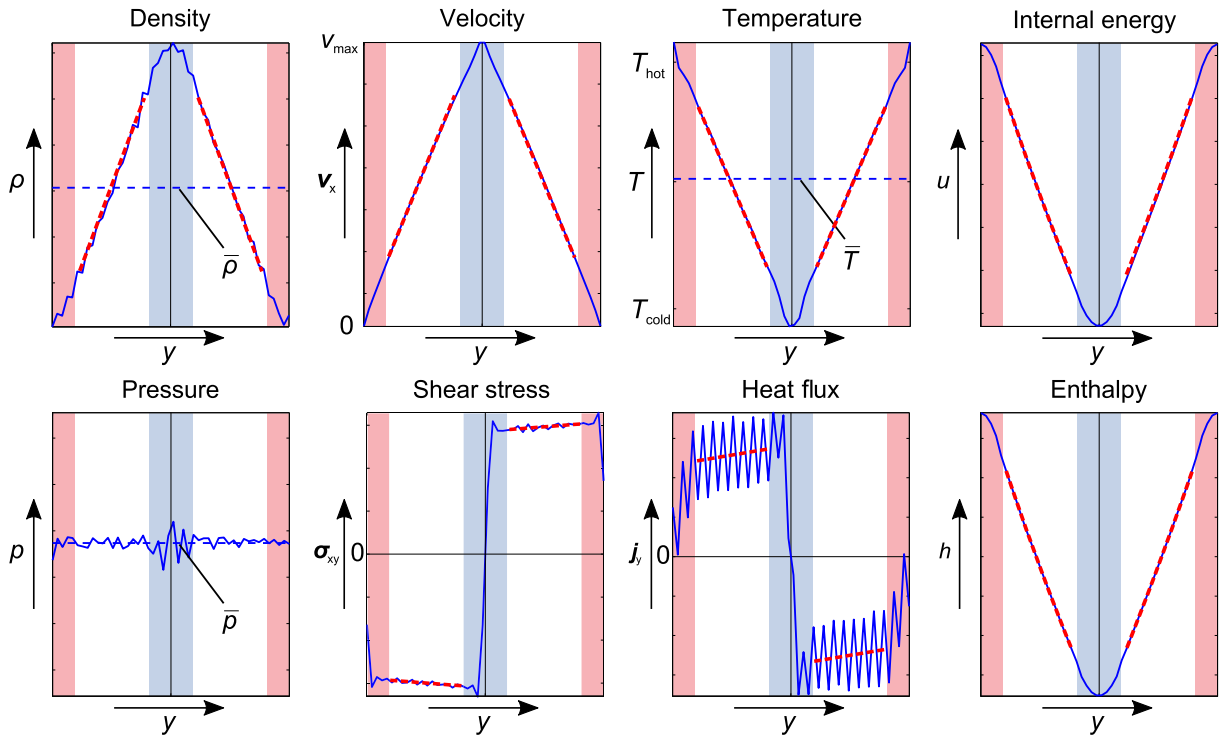

$$
\frac{\Delta X}{\bar{X}} \leq\left(\frac{\Delta J}{\bar{J}}+\frac{\Delta \Gamma}{\bar{\Gamma}}\right)
$$

where $X$ is the property for which the statistical uncertainty, i.e., relative approximation error $\Delta X / \bar{X}$, is determined and $J$ and $\Gamma$ are variables which are specified in Table I for the different properties $X$. In Eq. (8), the bars indicate the mean values and $\Delta$ indicates the empirical variance between the time-averaged simulation data and its corresponding regression fit.

The aforementioned error analysis was applied for each data point that was studied in the present work. The result of averaging the statistical uncertainties for a given set of data points, e.g., all data points in the liquid phase, is called "mean estimated uncertainty" (MEU).

The simulations were carried out with a substantially extended version of the MD code $l s 1$ mardyn. ${ }^{40}$

In the present work, the self-diffusivity in the $x$-, $y$-, and $z$ direction, i.e., $D_{x}, D_{y}$, and $D_{z}$, respectively, as well as the total selfdiffusion coefficient $D$ is distinguished and studied separately. In our previous works, ${ }^{8,9,27}$ only the total self-diffusion coefficient was studied. It is defined as the arithmetic average of the unidirectional self-diffusion coefficients,

$$
D=\frac{1}{3}\left(D_{x}+D_{y}+D_{z}\right)
$$

TABLE I. Specification of the variables $J$ and $\Gamma$ from Eq. (8) for the determination of the statistical uncertainties of the properties $X$ determined via postprocessing.

\begin{tabular}{lcc}
\hline \hline$X$ & $J$ & $\Gamma$ \\
\hline$\lambda$ & $\boldsymbol{j}_{y}$ & $\frac{\partial T}{\partial y}$ \\
$\eta$ & $\boldsymbol{\sigma}_{x y}$ & $\frac{\partial \boldsymbol{v}_{x}}{\partial y}$ \\
$c_{\mathrm{p}}$ & $\frac{\partial h}{\partial T}$ & $\frac{\partial T}{\partial y}$ \\
$\alpha_{\mathrm{p}}$ & $\frac{\partial(\ln \rho)}{\partial y}$ & $\frac{\partial T}{\partial y}$ \\
\hline \hline
\end{tabular}

\section{Structural analysis} point. $\dot{\gamma}_{\max }$.

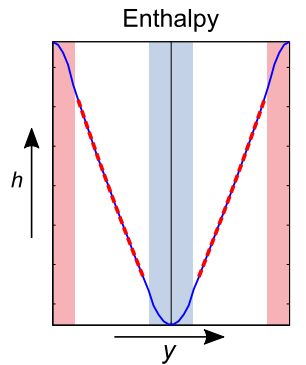

FIG. 2. Overview of observables measured in simulations using the twogradient method. The discretized results are depicted by the blue solid lines. The mean values of the density $\bar{\rho}$, temperature $\bar{T}$, and pressure $\bar{p}$ are depicted by the horizontal blue dashed lines. The fits are depicted by the red dashed lines. No data from the shaded regions were used for the regression.

In the present work, anisotropy has to be considered in the analysis of the structure of the fluid. Therefore, instead of the radial distribution function (RDF), ${ }^{3}$ which is insensitive for shear rates $\dot{\gamma} \lesssim 1.0$, the planar radial distribution function (pRDF) is used, which is described in more detail in Ref. 41 and in Appendix B, and shown to be sensitive for shear rates even below 1.0.

In Appendix B, the total pRDF $g_{k l}(r)$ is also defined. It is used as a measure for the average structure of the fluid and describes how particles arrange radially around each other in a certain distance $r$ in the $k l$-plane, where $k l$ stands for any combination of the coordinates $x, y$, and $z$. Under shear, this structure, i.e., basically the number of nearest neighbors, can change. This change of the structure is measured here by comparing the total pRDF of the fluid under shear to the total pRDF of the fluid in equilibrium and used for defining a criterion for the shear-rate sensitivity of a state

With the setup used in the present work, structural changes can only occur in the $x y$-plane, ${ }^{26}$ i.e., the only plane in which shear stresses occur. Thus, for a given state point $(T, \rho)$, the average structure in the $x y$-plane is quantified separately for each shear rate using $\tilde{g}_{x y}$ following a Kirkwood-Buff approach, ${ }^{42}$

$$
\tilde{g}_{x y}(\dot{\gamma})=\int_{0}^{\infty}\left|g_{x y}(r)-1\right| \mathrm{d} r .
$$

Using the quantity defined in Eq. (10), the shear-rate sensitivity factor $I_{x y}(\dot{\gamma})$ for the state point $(T, \rho)$ is determined from

$$
I_{x y}\left(\dot{\gamma}_{\max }\right)=\frac{1}{n-1} \sum_{\substack{i=1 \\ \dot{\gamma}_{i} \leq \dot{\gamma}_{\max }}}^{n}\left(1-\frac{\tilde{g}_{x y}\left(\dot{\gamma}_{i}\right)}{\tilde{g}_{x y}(0)}\right) .
$$

Equation (11) quantifies the mean deviation between the shear-rate affected values $\tilde{g}_{x y}\left(\dot{\gamma}_{i}\right)$ and its value at equilibrium conditions $\tilde{g}_{x y}(0)$ for a series of $n$ different shear rates $\dot{\gamma}_{i}$ which are not larger than 
As the statistical uncertainty of $\tilde{g}_{x y}(\dot{\gamma})$ is about $1 \%$ for all simulations from the present work, this value is used as a threshold for the shear-rate sensitivity. The data set is divided into two groups in the following: (1) Group A: When $I_{x y}\left(\dot{\gamma}_{\max }\right) \leq 1 \%$, no significant structural changes are observed and shear rates with $\dot{\gamma} \leq \dot{\gamma}_{\max }$ also do not lead to structural changes. It is shown below that this generally implies that also the fluid properties at that state point do not depend on the shear rate up to $\dot{\gamma}_{\max }$. The only exception is the self-diffusivity, as discussed below. (2) Group B: By contrast, when $I_{x y}\left(\dot{\gamma}_{\max }\right)>1 \%$, structural changes are observed which results in deviations of the fluid properties from their equilibrium values.

\section{OVERVIEW OF THE STUDY}

Figure 3 gives an overview of the 27 state points that were studied in the present work. A wide range of the liquid and the supercritical states is covered $(T \in[0.7,8.0], \rho \in[0.3,1.0])$. No state points with densities below $\rho=0.3$ were investigated as the twogradient method is not suited for such studies. ${ }^{27} p, u, h, c_{\mathrm{p}}, \alpha_{\mathrm{p}}, \lambda$, $\eta$, and $D$ were determined for all state points. At each state point, simulations with five different shear rates $\dot{\gamma} \in[0.1,1.0]$ were conducted. We have not studied shear rates below 0.1 as it is known that results for the LJTS fluid at such low shear rates do not differ from the equilibrium value $e^{8,9,27}$ and thus do not show a shear-rate dependence. $\lambda$ is the only property that is not discussed in detail in the following. On the one hand, in a previous study, ${ }^{27}$ it was shown that it does not depend significantly on the shear rate up to high shear rates. This is confirmed by the present study, cf. the supplementary material. On the other hand, the statistical uncertainty of $\lambda$ [determined as described with Eq. (8) or in detail in Ref. 27] was found to be in the order of the values of $\lambda$ for large shear rates.

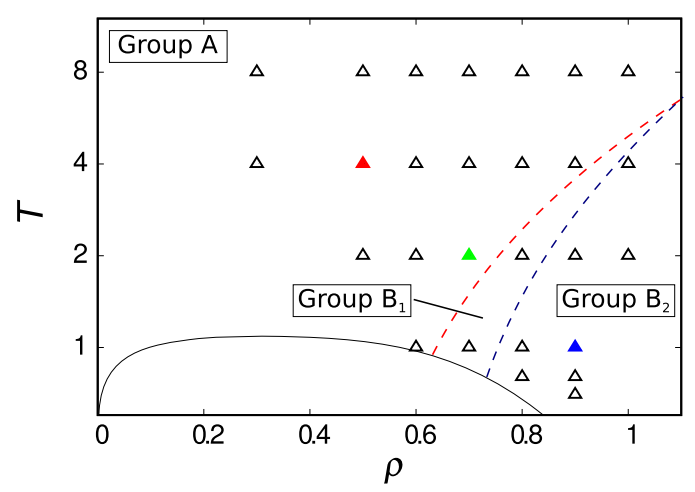

FIG. 3. Overview of the state points of the LJTS fluid that were studied in the present work. The state points are depicted by triangles in the $T, \rho$-space. State points where the triangles are filled (blue: $T=1.0, \rho=0.9$; green: $T=2.0, \rho=0.7$; and red: $T=4.0, \rho=0.5$ ) are discussed in detail in the following. The black line is the vapor-liquid coexistence line of the LJTS fluid which was determined with the PeTS equation of state. ${ }^{43}$ The dashed lines indicate thresholds of the shearrate sensitivity. For all states above the red dashed line (group A), no shear-rate dependence was observed in the present work (i.e., up to $\dot{\gamma}=1.0$ ). By contrast, an influence of the shear rate on the fluid properties for states below the red dashed line was observed for $\dot{\gamma}>0.5$ (group $\mathrm{B}_{1}$ ), and for states below the blue dashed line, the corresponding limit is $\dot{\gamma}>0.1$ (group $\mathrm{B}_{2}$ ).
This is due to the thermostatization which induces perturbations that lead to oscillations of the heat flux (cf. Fig. 2). However, these oscillations are steady, uniform, and evenly distributed around the red dashed line in Fig. 2, i.e., the linear regression fit. They hardly influence the fit itself (cf. the supplementary material) nor are any of the other profiles significantly affected by the oscillations of the heat flux profile. Thus, also the other second-order properties $\left(c_{\mathrm{p}}\right.$, $\alpha_{\mathrm{p}}$, and $\eta$ ) are not affected. These are strong indicators that the oscillations are only numerical artifacts of the calculation of the heat flux.

The numerical data of the full data set including $\lambda$ are given in the supplementary material. The mean estimated uncertainties of the other properties $\left(p, u, h, \eta, D, c_{\mathrm{p}}\right.$, and $\left.\alpha_{\mathrm{p}}\right)$ are $(1.5 \%, 1.6 \%, 1.7 \%, 2.5 \%$, $1.2 \%, 1.6 \%$, and $1.4 \%)$. They are well below the statistical uncertainty of the data reported in previous works on the shear-rate dependence of fluid properties. ${ }^{17,18}$

Figure 3 also indicates the shear-rate sensitivity of the state points studied in the present work. All states above the red dashed line (group A) are independent from the shear rate as long as $\dot{\gamma} \leq 1.0$. By contrast, an influence of the shear rate on the fluid properties for states below the red dashed line was observed for $\dot{\gamma}>0.5$ (group $\mathrm{B}_{1}$ ), and for states below the blue dashed line, the corresponding limit is $\dot{\gamma}>0.1$ (group $\mathrm{B}_{2}$ ). The criterion that was applied for the classification is the one described in Subsection II C. Note that for low values of $\dot{\gamma} \approx 0.1$, the basic idea behind this classification line could be connected to the Frenkel line.

\section{FLUID PROPERTIES, STRUCTURAL DATA, AND CORRELATIONS}

\section{A. Macroscopic fluid properties}

The numerical results of the entire data set are given in the supplementary material. The reported statistical uncertainties were determined as described above. Figures 4 and 5 show results of the present study of the shear-rate dependence of fluid properties for three different state points. The selected state points are marked with filled symbols in Fig. 3. Figure 4 shows the results of $p, \eta, u$, $h, c_{\mathrm{p}}$, and $\alpha_{\mathrm{p}}$. Figure 5 shows the results of the self-diffusion coefficients. The different state points are indicated by different colors. In addition to the results determined with the two-gradient method (empty symbols), also the equilibrium values of all properties are depicted by filled symbols at the axis of ordinates. For the thermal and caloric properties, the equilibrium data were determined using the equation of state of Thol et al. ${ }^{44}$ For the transport properties, the correlations from Ref. 8 were used. Besides the simulation data, also results from the correlations of the data that are described below are shown.

Macroscopic fluid properties are determined by the fluid's structure on the molecular scale. This also holds for the shear-rate dependence of these properties. Figures 4 and 5 show that for the state point with the lowest temperature and highest density $(T=1.0$, $\rho=0.9$ ), all studied properties depend on the shear rate. Even the simulation results for the shear rate $\dot{\gamma}=0.1$ deviate slightly from their equilibrium values (filled symbols), although the local structure of the fluid is not yet affected significantly. The deviations increase further with the increasing shear rate. By contrast, at the other two state points with higher temperature and lower density, for 

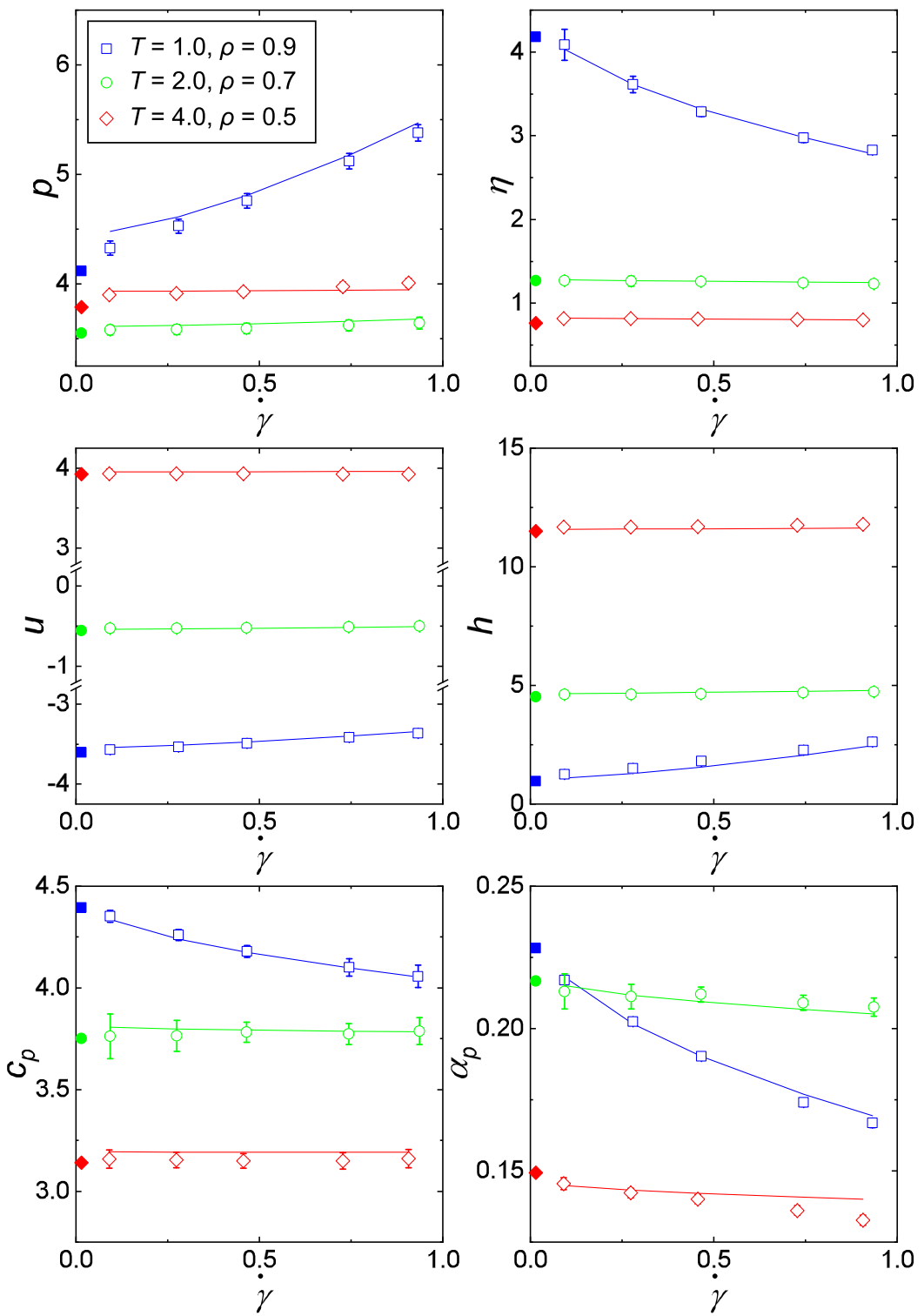

FIG. 4. Shear-rate dependence of thermal, caloric, and transport properties of the LJTS fluid. A comparison of data from the present work (empty symbols), equilibrium values 8,44 (filled symbols), and correlations from the present work (lines) for three state points, cf. legend. The estimated uncertainties of the results from the present work are depicted by the error bars which are mostly within the symbol size. most of the studied properties, no or only a weak influence of $j$ was observed and the results agree largely with the corresponding equilibrium values. A weak influence of $\dot{\gamma}$ is found for the thermal expansion coefficient $\alpha_{p}$ and the self-diffusion coefficient D. $\alpha_{p}$ decreases slightly with the increasing shear rate. The self-diffusivity shows an anisotropic behavior as is discussed further in the following.

\section{B. Local structure of the fluid}

In Fig. 6, the pRDFs for the $x y$ - (solid lines), $y z$ - (dashed lines), and $x z$-planes (dotted lines) are shown for two different state points with the same fluid density but different temperature and three different shear rates each. The pRDFs in the $y z$ - and $x z$-planes are not affected by the shear stresses for none of the shear rates applied in the present work. As supported by the literature, ${ }^{22,25,26}$ structural changes are only observed in the $x y$-plane. For the state point ( $T=0.8, \rho=0.9$ ), cf. left column of Fig. 6, shear-induced structural changes are observed. By contrast, for the state point $(T=8.0$, $\rho=0.9$ ), cf. right column of Fig. 6, no significant structural changes are observed.

Structural changes of the LJTS fluid under different shear rates are discussed by means of the examples shown in Fig. 6. There, the total pRDFs of the fluid for two characteristic state points are shown. The state point $(T=0.8, \rho=0.9)$ is influenced by the shearing, and the state point $(T=8.0, \rho=0.9)$ is not significantly affected. As already mentioned above, the pRDFs in the $y z$ - and $x z$-planes are not influenced for any of the state points and shear rates that are studied in the present work. 

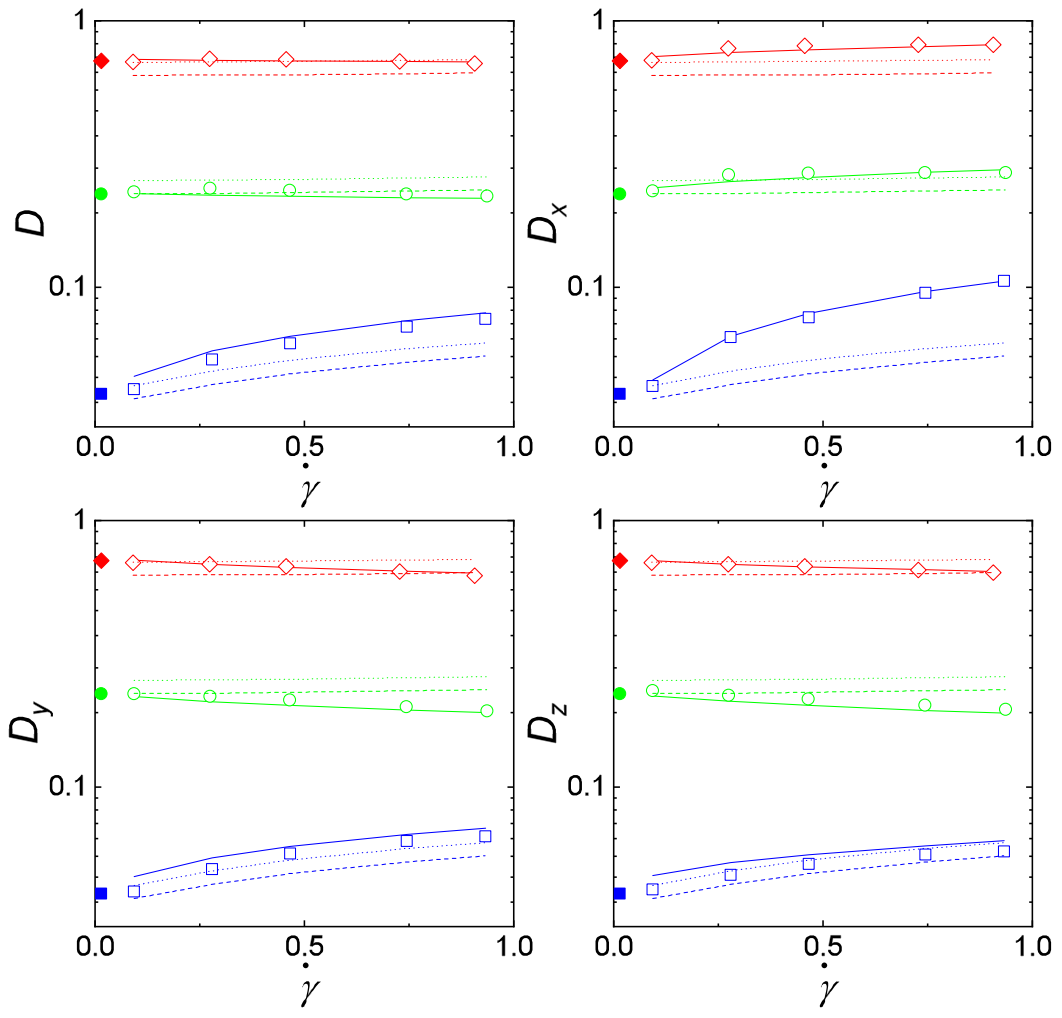

FIG. 5. Shear-rate dependence of the total self-diffusion coefficient $(D)$ and the unidirectional self-diffusion coefficients $\left(D_{x}, D_{y}\right.$, and $\left.D_{z}\right)$ of the LJTS fluid. A comparison of data from the present work (empty symbols), equilibrium values ${ }^{8}$ (filled symbols), correlations from the present work (solid lines), and theoretical predictions following the Stokes-Einstein relation from Ree et al..$^{45}$ (dashed lines) and $L i$ and $\mathrm{Chang}^{46}$ (dotted lines). Data are shown for three state points from which the colors and symbols are the same as in Fig. 4: ( $T=1.0, \rho=0.9)$-blue squares, $(T=$ 2.0, $\rho=0.7)$-green circles, and $(T=4.0, \rho=0.5)$-red diamonds. The estimated uncertainties of the results from the present work are within the symbol size.
Only the plots in the left column of Fig. 6 are discussed in detail in the following. Shear rates up to $\dot{\gamma}=0.1$ hardly affect the local structure of the fluid. However, with the increasing shear rate, the structure is increasingly disturbed. The amplitudes of the peaks of $g_{x y}(r)$ decrease, smear out, and approach $g_{x y}(r)=1.0$. The decrease in the first peak indicates a decrease in the number of nearest neighbors upon increasing the shear rate. Moreover, while for $\dot{\gamma}=0.1$ five peaks can be distinguished, for $\dot{\gamma}=1.0$ only three peaks are significant. These peaks correspond to layers of particles. These layers in the flat geometry are similar to the well-known shells of particles in radially symmetric problems (e.g., solvent molecules around an ion) which are commonly described by RDFs.

Structural changes were already reported in the literature. $^{11,22,23,25,26}$ Several authors ${ }^{22,25,26}$ found that the originally isotropic pRDF changes into an anisotropic biaxial ellipsoid, of which the long principal axis is aligned with $\pi / 4$ and the short principal axis is aligned with $3 \pi / 4$ in the $x y$-plane, cf. Fig. 8 of Ref. 26. These structural changes affect the fluid properties and may cause deviations from their equilibrium values. The present comprehensive study demonstrates that this effect is favored by states with low temperature and high density. A reason is that the local structure is governed by the repulsive part of the potential. This is particularly important at high densities which explains the increase in the shear-rate dependence with higher densities. High temperatures, on the other hand, lead to lower ordering, which results in a weaker shear-rate dependence.

In the present work, a characteristic number $I_{x y}\left(\dot{\gamma}_{\max }\right)$ is introduced that is suited to measure the influence of shearing with $\dot{\gamma} \leq \dot{\gamma}_{\max }$ on the structure of a fluid. Comparing this number with the statistical uncertainty of $\tilde{g}_{x y}(\dot{\gamma})$ enables to determine whether for a certain state point, a shear-rate dependence of fluid properties must be expected without actually measuring these properties. It is shown in the supplementary material that this criterion works well for almost all properties that were studied in the present work. As described above, the only exceptions are $\alpha_{p}$ and $D$.

\section{Shear-rate sensitivity}

From Eqs. (10) and (11), $I_{x y}\left(\dot{\gamma}_{\max }\right)$ was determined for each state point studied in the present work separately for the shear rates $\dot{\gamma}_{\max } \approx\{0.3,0.5,0.8,1.0\}$. The results are shown in Fig. 7 , where the dashed lines indicate the statistical uncertainty of $\tilde{g}_{x y}(\dot{\gamma})$, i.e., $1 \%$. Thus, all values of $I_{x y}$ above the dashed lines correspond to state points where the local structure of the fluid is significantly affected by the shear rate, i.e., group B in Fig. 3. These are most likely state points with low temperature and high density. As $\dot{\gamma}_{\max }$ increases, also states with lower density become affected.

\section{Correlations}

For some fluid properties, it has been shown in the literature that shear-rate effects can be described with simple functional relations. Such correlations have been reported for $p,{ }^{47,48} u,{ }^{47,48} \eta,{ }^{12,49}$ and $D .{ }^{11}$ All have in common that they consist of an equilibrium contribution (indicated with index 0 ) and a nonequilibrium contribution (indicated with index 1) and that both contributions depend on the density and temperature. 

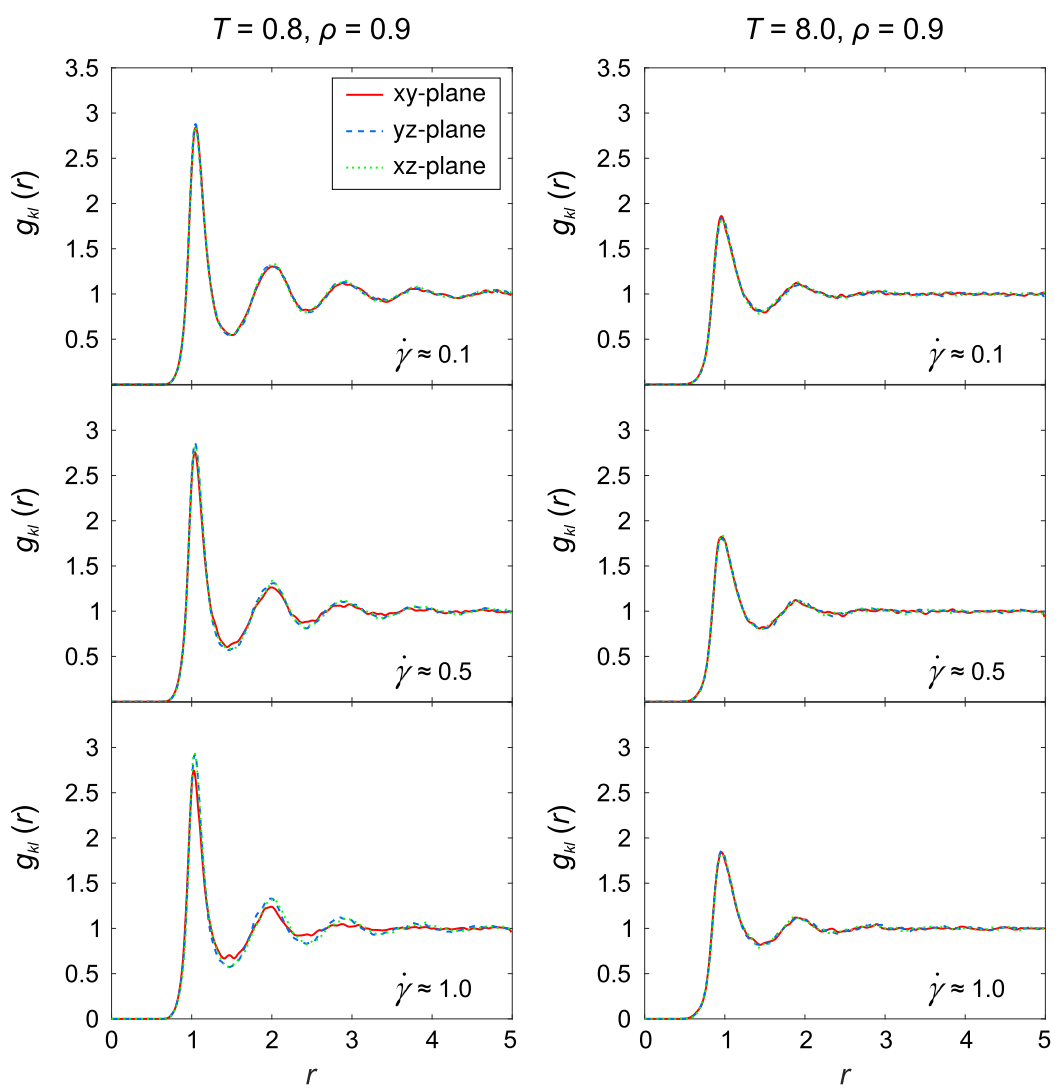

FIG. 6. Shear-rate dependence of the local structure of the fluid. The structure is determined via the total pRDF $g_{k l}(r)$ for the $x y$ - (red solid lines), $y z$ - (blue dashed lines), and $x z$-planes (green dotted lines). Two state points at equal density are shown for three different shear rates each.

Following Ref. 47, the generalized functional relation for $p$ and $u$ can be described with

$$
X(\rho, T, \dot{\gamma})=X_{0}(\rho, T)+X_{1}(\rho, T) \dot{\gamma}^{a},
$$

where the exponent $a$ depends on the density and temperature as suggested by Ref. 48 . In that reference, the following correlation for $a$ is reported:

$$
a(\rho, T)=\left\{\begin{array}{ll}
3.67+0.69 T-3.35 \rho, & \text { if } a \leq 2 \\
2, & \text { else }
\end{array} .\right.
$$

In the following, Eqs. (12) and (13) are also used to describe the shear-rate dependence of $h$.

Following Refs. 11, 12, and 49, the functional relation for $\eta$ and $D$ can be described with

$$
Y(\rho, T, \dot{\gamma})=Y_{0}(\rho, T)-Y_{1}(\rho, T) \dot{\gamma}^{\frac{1}{2}}
$$

Due to the similarity of the trends shown in Figs. 4 and 5, Eq. (14) is also used to describe the shear-rate dependence of $D_{x}, D_{y}, D_{z}, c_{\mathrm{p}}$, and $\alpha_{\mathrm{p}}$ in the present work.

Based on the full data set of the present study, the parameters $X_{0}, X_{1}, Y_{0}$, and $Y_{1}$ of Eqs. (12) and (14) for all aforementioned fluid properties were determined by least squares regressions. Note that Eqs. (12) and (14) were not adjusted to the equilibrium values (filled symbols in Figs. 4 and 5). For all parameters $X_{0}, X_{1}, Y_{0}$, and $Y_{1}$, correlations were developed with the software Eureqa $a^{50}$ using the error metric "mean squared error." Eureqa provides empirical correlations of given data in an automated way. The correlations suggested by Eureqa were checked for smoothness and unphysical artifacts. All correlations that are published here passed these tests. They cannot replace physical models but may be useful for applications. The correlations are listed in Appendix C. They should only be used in the range in which data were available for the fit.

\section{E. Comparison of the correlations with literature data}

The shear rates that were studied in the present work are very high. No experimental data for such high shear rates are available in the literature. Hence, for a comparison, only MD data on LJ-type fluids from the literature were used. ${ }^{16-18}$ These data are compared here to the correlations that were obtained in the present work using the simulation data for the LJTS fluid. It is known from a previous study of our group ${ }^{8}$ that the truncation and shifting of the LJ potential has only little influence on the viscosity as long as the thermodynamic state is not too close to the critical point. For such states, it is also known that the truncation has only little influence on $D^{8}$ so that one may expect that this direct comparison of results for different LJtype fluids yields results that do not differ strongly. Results of the pressure and internal energy could not be compared with the results 

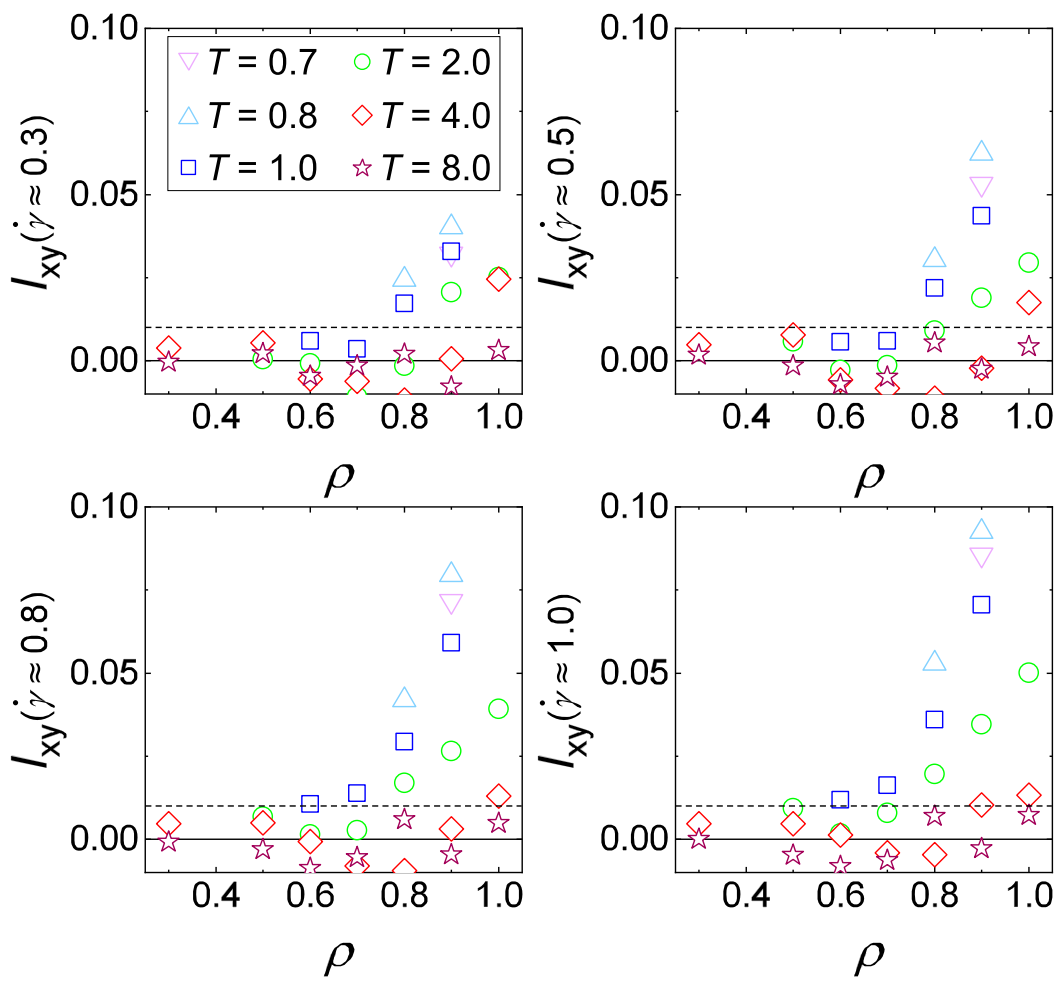

FIG. 7. Dependence of the shear-rate sensitivity factor $I_{x y}\left(\dot{\gamma}_{\max }\right)$ [cf. Eq. (11)] on the temperature and density. Dashed lines indicate the statistical uncertainty of $\tilde{g}_{x y}(\dot{\gamma})$ which is about $1 \%$. The local structure of the fluid of states above the dashed lines is significantly affected. from the present work as those depend strongly on the type of LJ potential.

The comparison is restricted to the range of the validity of the correlations from the present work, i.e., $T \in[0.7,8.0], \rho \in[0.3,1.0]$, and $\dot{\gamma} \in[0.1,1.0]$. The results are shown in deviation plots in Fig. 8. The literature data on $\eta$ from Heyes ${ }^{18}$ and Ahmed and Sadus ${ }^{16}$ agree very well with the correlations over the whole range of thermodynamic states that was considered in the present work. They scatter mainly within a band of $\pm 10 \%$. However, the older data on $\eta$ from Heyes $^{17}$ deviate more than $10 \%$ for $\eta \lesssim 3$. The literature data for $D^{17,18}$ show less good agreement with the correlation. The correlation tends to overestimate the literature data, and deviations of more than $10 \%$ occur more frequently.

A more detailed quantitative comparison of the correlations with literature data is given in the supplementary material. The correlations from the present were also compared to the data from Ref. 1. However, the corresponding data set is not shown in Fig. 8 as the results scatter significantly due to the small system sizes used there.

\section{F. Anisotropy of the self-diffusivity}

The anisotropy of the self-diffusivity is shown in Fig. 5. Besides data on the total self-diffusion coefficient $D$, also the unidirectional self-diffusion coefficients $D_{x}, D_{y}$, and $D_{z}$ are shown. Different effects are observed. For the state for which a shear-rate dependence of the structure is observed (blue squares, group B), D increases strongly with the increasing shear rate. For the other two states (green circles, group A) and (red diamonds, group A), D is constant or even decreases slightly. Since $D$ is the arithmetic average of the unidirectional self-diffusion coefficients [cf. Eq. (9)], all contributions are separately discussed in the following.

For all state points depicted in Fig. $5, D_{x}$ increases with the increasing shear rate and the relative growth of $D_{x}$ is the largest for the state in group B. The findings are different for $D_{y}$ and $D_{z}$. There, the unidirectional diffusivity is enhanced for the state in group B, while it is decreasing for states in group A. Moreover, $D_{y}$ and $D_{z}$ have similar values for states in group A, while $D_{y}$ is always larger than $D_{z}$ for the state in group B. In all cases, the influence of the shear rate is larger for the state in group B than for the states in group A.

These findings are in agreement with those from several authors $^{15,52,53}$ and can be explained as a superposition of four different phenomena: (1) The shearing in the $x$-direction generally facilitates the self-diffusivity in the $x$-direction. The shear stresses exerted by the nearest neighbor particles reduce the energy barrier that must be overcome by a particle to diffuse. This has also been shown theoretically. ${ }^{54}$ This effect is independent of any structural changes such that $D_{x}$ increases with the increasing shear rate for all state points. (2) As discussed before, for the states in group B, a sufficient shearing leads to a deformation of the shell structure of the nearest neighbor particles such that particles can easily escape from this shell. As the structural changes appear in the $x y$-plane only, only the selfdiffusion coefficients $D_{x}$ and $D_{y}$ are enhanced by this phenomenon. (3) As was shown in the literature, ${ }^{15,55}$ the unidirectional contributions of the random kinetic energy can become anisotropic under shear as well. The $y$ - and $z$-contributions decrease at the expense of an increase in the contribution in the $x$-direction. This effect is 

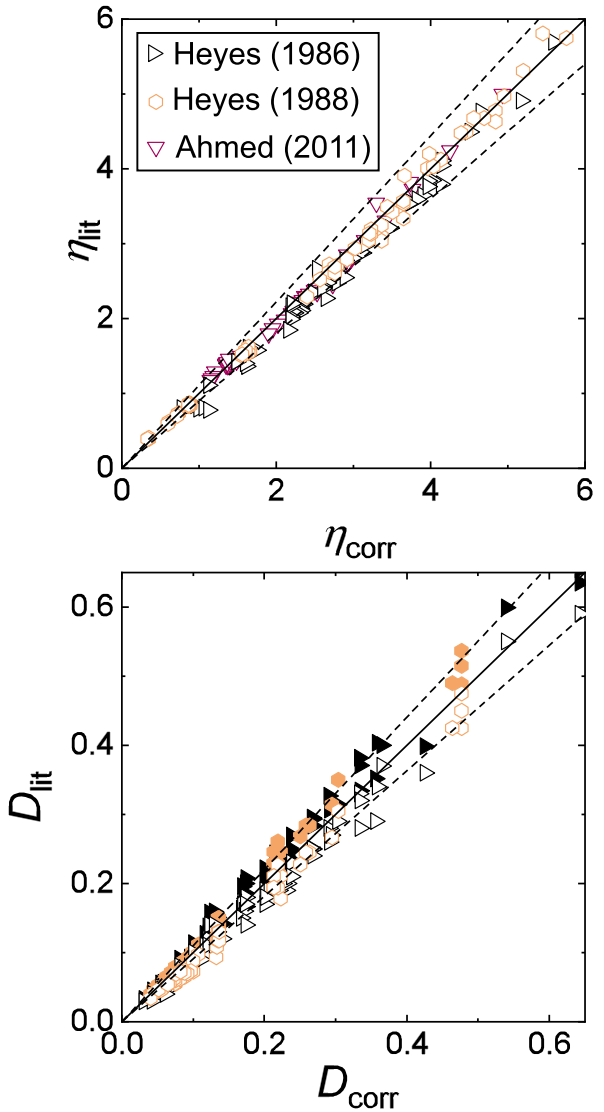

FIG. 8. Deviation plots comparing literature data on $\eta$ and $D$ of the LJT fluid with the correlations developed in the present work for the LJTS fluid. The investigated state points are in the liquid and the supercritical regime with shear rates in the range of $\dot{\gamma} \in[0.1,1.0]$. The original literature data are depicted by empty symbols (see legend). For $D$, also results are shown that were obtained by correcting the literature data for finite-size effects as suggested by Yeh and Hummer ${ }^{37}$ (filled symbols). The dashed lines indicate a band of $\pm 10 \%$ around the solid lines.

increasingly significant at high shear rates and reduces $D_{y}$ and $D_{z}$, which is especially apparent for states for which the structure does not depend on the shear rate, i.e., group A. (4) For states for which the structure is affected, i.e., group B, an increasing shear rate causes a reduction of the shear viscosity. Thus, besides $D_{x}$ and $D_{y}$, also $D_{z}$ is enhanced.

\section{G. Relation between shear viscosity and self-diffusion coefficient}

Based on the Stokes-Einstein relation ${ }^{31}$ and Eyring's kinetic theory, ${ }^{56}$ different theories ${ }^{45,46}$ have been developed to relate the shear viscosity with the self-diffusion coefficient. They were derived for equilibrium conditions in bulk fluids. Giannakopoulos et al. ${ }^{5}$ reported that they are also valid for confined fluids in nanochannels. It is discussed here to which extent these theories are applicable for describing properties of fluids under shear, where the self-diffusivity is anisotropic, cf. Sec. IV.
The aforementioned theories can be generalized to

$$
D=\frac{T}{\xi \eta} \sqrt[3]{\rho}
$$

where the only difference between the theories is the value of $\xi$, which can be interpreted as a weighting factor that is proportional to the number of nearest neighbors. The most common values of $\xi$ that were reported in the literature are $\xi=5.6$ from Ref. $45, \xi=6.0$ from Ref. 46 , and $\xi=2 \pi$ from Ref. 46 .

The simulation results of $D, D_{x}, D_{y}$, and $D_{z}$ from the present work are compared to the values determined from Eq. (15). The comparison is shown for three exemplary state points in Fig. 5. There, the values determined from Eq. (15) for $\xi=5.6$ and $\xi=2 \pi$ are depicted by dashed and dotted lines, respectively. In addition, a detailed quantitative analysis is given in the supplementary material.

The main results from these data are (1) close to the equilibrium $(\dot{\gamma}=0.1)$, where the self-diffusivity is isotropic, Eq. (15) with $\xi=6.0$ agrees best with the data from the present work. This is the case for both states of group A and states of group B. (2) For states of group A, $D$ can be predicted by Eq. (15) also for high shear rates $(\dot{\gamma}=1.0)$. In this case, best agreement is observed for $\xi=2 \pi$. (3) For states in group B, the theories strongly underestimate $D$ at high shear rates. The agreement between both improves with decreasing $\xi$ indicating that the number of nearest neighbors decreases under shear (cf. Fig. 6).

\section{CONCLUSION}

In previous studies of our group, ${ }^{8,27}$ the two-gradient method was developed and used for the determination of equilibrium properties of the Lennard-Jones fluid truncated and shifted at the cut-off radius $r_{\mathrm{c}}^{*}=2.5 \sigma$ (LJTS fluid). In the present study, it is shown that this method is also applicable for studying the influence of shear stresses on the properties pressure $p$, internal energy $u$, enthalpy $h$, isobaric heat capacity $c_{\mathrm{p}}$, thermal expansion coefficient $\alpha_{\mathrm{p}}$, shear vis$\operatorname{cosity} \eta$, and self-diffusion coefficient $D$. Due to the superposition of a temperature gradient with a velocity gradient, all aforementioned properties were obtained from a single simulation run, which is a significant advantage of the two-gradient method. Also, for $\lambda$, results were obtained, which are however not useful as they are subject to large uncertainties. By contrast, the uncertainties of the data for the other properties are not substantially larger than in typical studies of fluids in equilibrium.

A comprehensive study was conducted for the LJTS fluid. Data on the aforementioned properties were determined for 27 state points which cover a wide range of thermodynamic states $(T \in[0.7$, $8.0]$ and $\rho \in[0.3,1.0])$. At each state point, the shear rate was varied in the range $\dot{\gamma} \in[0.1,1.0]$. Thus, the literature data on the shear-rate dependence of properties of LJ-type fluids were largely extended. Based on the data, simple empirical correlations were developed for all properties. The correlations describe the simulation results very well. For $\eta$ and $D$, the results from the correlations were compared to the literature data. A good agreement was observed for $\eta$. For $D$, the literature data that were determined using small systems had to be corrected for finite-size effects. After this correction, also for $D$, a good agreement was found. 
The local structure of the fluid was studied using planar radial distribution functions (pRDFs). It was found that shear stresses change the local structure of the LJTS fluid when the shear rate exceeds a certain threshold. The changes are only observed in the plane that is exposed to shear stresses. The structural changes depend on the thermodynamic state of the fluid. They are favored by states with low temperatures and high densities. Based on the pRDFs, a criterion for the shear-rate sensitivity of a given state point was developed. Furthermore, it was shown that the structural changes of the fluid as well as the shear flow lead to anisotropic effects of the self-diffusivity. Finally, the results of the different contributions of the self-diffusion coefficient were compared to theories that are based on the Stokes-Einstein relation. It was found that these theories are applicable for small shear rates only. However, with small adaptations, they can also be used to predict the behavior of fluids under shear.

The present study has proven that the two-gradient method is suited for efficient calculations of thermodynamic properties under shear and can be used to study structural phenomena as well. The results from the present work support that there is a shear-rate dependence also for very simple, monatomic fluids. Due to the wide range of thermodynamic states and shear rates investigated, it was also shown when a significant shear-rate dependence has to be expected for simple fluids. The corresponding shear rates are usually orders of magnitudes larger than technically relevant shear rates. The data from the present work can, however, be used for developing general models of the shear-rate dependence of fluids. It is therefore useful as a reference, even when considering more complex fluids.

\section{SUPPLEMENTARY MATERIAL}

See supplementary material for the numerical results and the statistical uncertainties of all properties for each combination of $T$, $\rho$, and $\dot{\gamma}$ studied in the present work. There, beside the thermal, caloric, and transport properties, also data for the shear-rate sensitivity factor $I_{x y}\left(\dot{\gamma}_{\max }\right)$ are given. In addition, supplementary material regarding the evaluation of the correlations from the present work is given.

\section{ACKNOWLEDGMENTS}

The project was funded by the Deutsche Forschungsgemeinschaft (DFG, the German Research Foundation)-No. 252408385IRTG 2057 Physical Modeling for Virtual Manufacturing Systems and Processes as well as by the Federal Ministry of Education and Research (BMBF, Germany)-No. 01IH16008-Task-based load balancing and auto-tuning in particle simulations (TaLPas). The simulations were carried out on the elwe at Regional University Computing Center Kaiserslautern (RHRK) under the grant TUKLTLMV as well as on the HAZELHEN at the Supercomputing Centre Stuttgart (HLRS) under the grant "Molecular Modelling of Hydrogen Bonding Fluids" (MMHBF2). The present research was conducted under the auspices of the Boltzmann-Zuse Society for Computational Molecular Engineering (BZS).

There are no conflicts to declare.

\section{APPENDIX A: REDUCED UNITS}

TABLE II. Definition of the physical quantities in reduced units (plain). The corresponding observable carrying a dimension is marked by the asterisk. $M$ is the mass of a single particle. $k_{\mathrm{B}}$ is Boltzmann's constant.

\begin{tabular}{lc}
\hline \hline Length & $L=\frac{L^{*}}{\sigma}$ \\
Time & $\tau=\frac{\tau^{*}}{\sigma \sqrt{M / \varepsilon}}$ \\
Mass & $m=\frac{m^{*}}{M}$ \\
Velocity & $\boldsymbol{v}_{k}=\frac{\boldsymbol{v}_{k}^{*}}{\sqrt{\varepsilon / M}}$ \\
Shear rate & $\dot{\gamma}=\frac{\dot{j}^{*}}{\sqrt{\varepsilon / M} / \sigma}$ \\
Density & $\rho=\rho^{*} \sigma^{3}$ \\
Temperature & $T=\frac{T^{*}}{\varepsilon / k_{\mathrm{B}}}$ \\
Pressure & $p=\frac{p^{*}}{\varepsilon / \sigma^{3}}$ \\
Internal energy & $u=\frac{u^{*}}{\varepsilon}$ \\
Enthalpy & $h=\frac{h^{*}}{\varepsilon}$ \\
Shear viscosity & $\eta=\frac{\eta^{*}}{\sqrt{\varepsilon M} / \sigma^{2}}$ \\
Self-diffusion coefficient & $D=\frac{D^{*}}{\sigma \sqrt{\varepsilon / M}}$ \\
Isobaric heat capacity & $c_{\mathrm{p}}=\frac{c_{\mathrm{p}}^{*}}{k_{\mathrm{B}}}$ \\
Thermal expansion coefficient & $\alpha_{\mathrm{p}}=\frac{\alpha_{\mathrm{p}}^{*}}{k_{\mathrm{B}} / \varepsilon}$ \\
\hline \hline
\end{tabular}

\section{APPENDIX B: PLANAR RADIAL DISTRIBUTION FUNCTION}

Compared to the RDF, the main difference is that the structure of the system is not evaluated in a three-dimensional spherical space but only in a two-dimensional circular space. The simulation volume was divided in planelike slabs with a thickness of $d=0.7$ each. For each slab, a pRDF is determined. The total pRDF results from averaging these pRDFs over all slabs in the system. Following Ref. 41 , the procedure of the determination of the total pRDF is defined as

$$
g_{k l}(r)=\left\langle\frac{N_{\text {slab }}(r, \Delta r)}{N_{\text {slab }} \rho_{\text {slab }} \pi d\left(\left(r+\frac{1}{2} \Delta r\right)^{2}-\left(r-\frac{1}{2} \Delta r\right)^{2}\right)}\right\rangle .
$$

Here, $g_{k l}(r)$ is the total pRDF in the $k l$-plane, where $k l$ stands for any combination of the coordinates $x, y$, and $z . N_{\text {slab }}(r, \Delta r)$ is the number of particles in a ring with the mean radius $r$ and the width $\Delta r$ within a single slab. $N_{\text {slab }}$ is the total number of particles in the slab, and $\rho_{\text {slab }}$ is the mean density in the slab. The residual term in the denominator, i.e., $\pi d\left((\cdot)^{2}-(\cdot)^{2}\right)$, is the volume of the ring in the slab. The angular brackets indicate a spatial averaging over all slabs in the systems as well as an averaging over time.

\section{APPENDIX C: CORRELATIONS}

$$
\begin{aligned}
p_{0}= & 42.1001 \rho^{3}-43.99 \rho^{2}+8.481+2.402 \rho^{3} T+5.691 \rho^{3} \ln (T) \\
& +1.373 \rho T-1.8740 \rho^{-1}, \\
p_{1}= & -0.375922 \rho^{3} \ln \left(1.926 \rho^{-1}+5.924 \rho \ln (\rho)-1.926 \ln (\rho)\right) \\
& +2.69514 \rho^{7} T^{-1}-0.073208 \rho T^{-1}+0.073208,
\end{aligned}
$$




$$
\begin{aligned}
u_{0}= & 7.35153 \rho^{3}-12.2476 \rho^{2}-0.000423915 T^{3}+0.932114 \rho^{3} \ln (T) \\
& +0.561188 \rho^{2} T+1.61368 T-1.05479, \\
u_{1}= & -0.0599699-0.000261461 T^{3}+0.00276476 T^{2} \\
& -0.0642942 / T^{2}+0.378578 /\left(-\rho^{5}-2.544 \rho T\right. \\
& +3.362 T+0.5936), \\
h_{0}= & 70.1479 \rho^{2}-135.119 \rho+26.674 \ln (\rho)+8.19408 \rho^{3} \ln (T) \\
& +T(3.91965 \rho-0.280831 \ln (\rho)+1.65201)+63.4419, \\
h_{1}= & -0.259117 \exp \left(\rho^{3}\right) \rho^{2}-0.00154508 T^{2}+3.78956 \rho^{7} T^{-1} \\
& +0.0478455 \rho^{3} T+0.14519, \\
\eta_{0}= & -0.0545154+1.71528 \rho+7.95004 \rho^{8} T^{-1}+0.31938 \rho T \\
& +0.0585714 T \rho^{-1}-0.245255 T-0.406993 T^{-1}, \\
\eta_{1}= & 0.0385+0.000005285 T \exp (9.229 \rho)+(0.266-0.4512 \rho) T^{-3} \\
& +0.0008492 \rho^{3} \exp (9.229 \rho) /\left(0.1484+T^{2}\right) \\
& -0.00005179 \exp (9.229 \rho),
\end{aligned}
$$$$
D_{0}=-2.90184 \rho^{2}+\ln (\rho)\left(5.33686 \rho+0.0141243 T^{2}+0.257997\right)
$$$$
+\left(0.228609 \rho^{2}-0.632747 \rho+0.457217\right) T+2.84286,
$$

$D_{1}=-0.4964 \rho-0.2229 \rho^{-1}+0.03613 \rho^{-2}+0.05419 T \rho$ $-0.002305 T^{2} \rho-0.00533 T^{2}+0.00004373 T^{4}+0.557, \quad(\mathrm{C} 10)$

$D_{\mathrm{x}, 0}=-0.0272187 \exp (\rho) \rho^{2}+0.0858858 \rho^{-1}-0.00768892 T^{2} \rho^{-1}$

$$
+0.00615034 T^{2}+1.88292 \rho^{2} T-1.44005 \exp (\rho) T
$$$$
+2.10087 T-0.106088 \text {, }
$$

$$
D_{\mathrm{x}, 1}=-0.71009 \rho^{2}+0.884338 \rho+0.000473426 T^{3}-0.00927007 \rho T^{2}
$$$$
+0.07475 \rho^{2} T-0.0172065 T-0.348397 \text {, }
$$

$D_{\mathrm{y}, 0}=0.0206414 T^{2} \rho-0.0200619 T^{2}-0.295134 T \rho+0.338695 T$

$$
-0.566789 \rho^{4}+3.19412 \rho^{2}-4.06534 \rho+1.40673,
$$

$D_{\mathrm{y}, 1}=-0.0454464(T \rho-0.884294 T-1.54773 \ln (T+0.6194)$ $-5.24637 \rho+7.14914)+0.0580726(T-7.62438) \ln (\rho)$,

$$
\begin{aligned}
D_{\mathrm{z}, 0}= & 1.365+0.3312 T+3.164 \rho^{2}+0.01934 T^{2} \rho-3.975 \rho \\
& -0.01909 T^{2}-0.2839 T \rho-0.5874 \rho^{4}, \\
D_{\mathrm{z}, 1}= & -0.2454 \rho-0.0006982 T-0.04107 \rho^{2}-0.002187 T^{2} \\
& +0.2023+0.03329 T \rho, \\
c_{\mathrm{p}, 0}= & -1.277 T \exp \left(\left(0.7305 T^{-1}-0.8138\right) \rho^{-1}\right)+T^{0.8628} \rho^{0.8974} \\
& -\rho^{-0.8593}+0.0141 \rho \exp \left((-14.94 \rho+15.03) T^{-1}\right) \\
& +5.592(T \rho)^{-0.272}, \\
& c_{\mathrm{p}, 1}=3.31024 \exp \left(\rho\left(2.615 \rho^{3}-4.179\right) T\right),
\end{aligned}
$$

$$
\begin{aligned}
\alpha_{\mathrm{p}, 0}= & \rho\left(-0.166197+0.00180039 T^{2}-0.245835 T^{-2}\right. \\
& +198.343 /(500 \rho-598+603 \rho T))-0.0165696 T+0.174121,
\end{aligned}
$$

$$
\begin{aligned}
\alpha_{\mathrm{p}, 1}= & -0.0119304+0.00830735 \rho^{-1} \\
& +(70.2238 T) /\left(\exp \left(0.3862 \rho^{2} T^{4}\right)\right. \\
& \left.+11.03 \rho^{2} T \exp \left(11.03 \rho^{2} T\right)\right)+0.0837729 \rho T^{-2} .
\end{aligned}
$$

\section{REFERENCES}

'B. V. Raghavan and M. Ostoja-Starzewski, "Shear-thinning of molecular fluids in Couette flow," Phys. Fluids 29, 023103 (2017).

${ }^{2}$ R. I. Tanner, "Review article: Aspects of non-colloidal suspension rheology," Phys. Fluids 30, 101301 (2018).

${ }^{3}$ M. P. Allen and D. J. Tildesley, Computer Simulation of Liquids (Clarendon Press, Oxford Science Publications, Oxford, 1989).

${ }^{4} \mathrm{~A}$. Trokhymchuk and J. Alejandre, "Computer simulations of liquid/vapor interface in Lennard-Jones fluids: Some questions and answers," J. Chem. Phys. 111, 8510-8523 (1999).

${ }^{5}$ V. Baidakov, G. Chernykh, and S. Protsenko, "Effect of the cut-off radius of the intermolecular potential on phase equilibrium and surface tension in LennardJones systems," Chem. Phys. Lett. 321, 315-320 (2000).

${ }^{6} \mathrm{~J}$. López-Lemus and J. Alejandre, "Thermodynamic and transport properties of simple fluids using lattice sums: Bulk phases and liquid-vapour interface," Mol. Phys. 100, 2983-2992 (2002).

${ }^{7}$ G. Rutkai, M. Thol, R. Span, and J. Vrabec, "How well does the Lennard-Jones potential represent the thermodynamic properties of noble gases?," Mol. Phys. 115, 1104-1121 (2017).

${ }^{8}$ M. P. Lautenschlaeger and H. Hasse, "Transport properties of the Lennard-Jones truncated and shifted model fluid from non-equilibrium molecular dynamics calculations," Fluid Phase Equilib. 482, 38-47 (2019).

${ }^{9}$ M. P. Lautenschlaeger and H. Hasse, "Thermal and caloric properties of fluids from non-equilibrium molecular dynamics simulations using the two-gradient method," J. Chem. Phys. 149, 244106 (2018).

${ }^{10}$ D. J. Evans and R. Watts, "Shear-dependent viscosity in simple fluids," Chem. Phys. 48, 321-327 (1980).

${ }^{11}$ D. M. Heyes, "Shear thinning and thickening of the Lennard-Jones liquid. A molecular dynamics study," J. Chem. Soc., Faraday Trans. 2 82, 1365 (1986).

${ }^{12}$ D. J. Evans, "Rheological properties of simple fluids by computer simulation," Phys. Rev. A 23, 1988-1997 (1981).

${ }^{13}$ H. J. M. Hanley and D. J. Evans, "A thermodynamics for a system under shear," J. Chem. Phys. 76, 3225-3232 (1982).

${ }^{14}$ D. M. Heyes, J. J. Kim, C. J. Montrose, and T. A. Litovitz, "Time dependent nonlinear shear stress effects in simple liquids: A molecular dynamics study," $\mathrm{J}$. Chem. Phys. 73, 3987-3996 (1980).

${ }^{15}$ S. Sarman, D. J. Evans, and A. Baranyai, "Mutual and self-diffusion in fluids undergoing strong shear," Phys. Rev. A 46, 893-902 (1992).

${ }^{16}$ A. Ahmed and R. J. Sadus, "Nonequilibrium equation of state for Lennard-Jones fluids and the calculation of strain-rate dependent shear viscosity," AIChE J. 57, 250-258 (2011).

${ }^{17} \mathrm{D}$. Heyes, "The molecular dynamics study of shear thinning of the LennardJones fluid," Chem. Phys. 109, 47-65 (1986).

${ }^{18}$ D. M. Heyes, "Viscosity and self-diffusion of simple liquids. Hard-sphere treatment of molecular dynamics data," J. Chem. Soc., Faraday Trans. 2 83, 1985 (1987).

${ }^{19}$ P. T. Cummings, B. Y. Wang, D. J. Evans, and K. J. Fraser, "Nonequilibrium molecular dynamics calculation of self-diffusion in a non-Newtonian fluid subject to a Couette strain field," J. Chem. Phys. 94, 2149-2158 (1991).

${ }^{20}$ J. Delhommelle, J. Petravic, and D. J. Evans, "Non-Newtonian behavior in simple fluids," J. Chem. Phys. 120, 6117-6123 (2004). 
${ }^{21}$ R.-y. Dong, B.-y. Cao, H.-m. Yun, and B.-m. Chen, "Study on non-newtonian behaviors of Lennard-Jones fluids via molecular dynamics simulations," Chin. J. Chem. Phys. 29, 754-760 (2016).

${ }^{22}$ H. J. M. Hanley, J. C. Rainwater, and S. Hess, "Shear-induced angular dependence of the liquid pair correlation function," Phys. Rev. A 36, 1795-1802 (1987).

${ }^{23}$ S. Hess and H. J. M. Hanley, "Distortion of the structure of a simple fluid," Phys. Rev. A 25, 1801-1804 (1982).

${ }^{24}$ W. P. Krekelberg, V. Ganesan, and T. M. Truskett, "Shear-rate-dependent structural order and viscosity of a fluid with short-range attractions," Phys. Rev. E: Stat, Nonlinear, Soft Matter Phys. 78(1), 010201 (2008).

${ }^{25} \mathrm{~T}$. Yamaguchi, "Stress-structure coupling and nonlinear rheology of LennardJones liquid," J. Chem. Phys. 148, 234507 (2018).

${ }^{26} \mathrm{X}$. Yong and L. T. Zhang, "Nanoscale simple-fluid behavior under steady shear," Phys. Rev. E: Stat., Nonlinear, Soft Matter Phys. 85, 051202 (2012).

${ }^{27}$ M. P. Lautenschlaeger, M. Horsch, and H. Hasse, "Simultaneous determination of thermal conductivity and shear viscosity using two-gradient non-equilibrium molecular dynamics simulations," Mol. Phys. 117, 189-199 (2019).

${ }^{28} \mathrm{P}$. Wirnsberger, D. Frenkel, and C. Dellago, "An enhanced version of the heat exchange algorithm with excellent energy conservation properties," J. Chem. Phys. 143, 124104 (2015).

${ }^{29}$ D. J. Evans and G. P. Morriss, "Shear thickening and turbulence in simple fluids," Phys. Rev. Lett. 56, 2172-2175 (1986).

${ }^{30}$ M. P. Allen and F. Schmid, "A thermostat for molecular dynamics of complex fluids," Mol. Simul. 33, 21-26 (2007).

${ }^{31}$ A. Einstein, "Über die von der molekularkinetischen theorie der Wärme geforderte bewegung von in ruhenden flüssigkeiten suspendierten teilchen," Ann. Phys. 322, 549-560 (1905).

${ }^{32}$ J. Vrabec, G. K. Kedia, G. Fuchs, and H. Hasse, "Comprehensive study of the vapour-liquid coexistence of the truncated and shifted Lennard-Jones fluid including planar and spherical interface properties," Mol. Phys. 104, 1509-1527 (2006).

${ }^{33}$ K. Binder, J. Horbach, W. Kob, W. Paul, and F. Varnik, "Molecular dynamics simulations," J. Phys.: Condens. Matter 16, S429-S453 (2004).

${ }^{34} \mathrm{M}$. Bugel and G. Galliero, "Thermal conductivity of the Lennard-Jones fluid: An empirical correlation," Chem. Phys. 352, 249-257 (2008).

${ }^{35} \mathrm{~J}$. Nichele, I. Borges, A. B. Oliveira, and L. S. Alves, "Molecular dynamics simulations of momentum and thermal diffusion properties of near-critical argon along isobars," J. Supercrit. Fluids 114, 46-54 (2016).

${ }^{36}$ O. A. Moultos, Y. Zhang, I. N. Tsimpanogiannis, I. G. Economou, and E. J. Maginn, "System-size corrections for self-diffusion coefficients calculated from molecular dynamics simulations: The case of $\mathrm{CO}_{2}, \mathrm{n}$-alkanes, and poly(ethylene glycol) dimethyl ethers," J. Chem. Phys. 145, 074109 (2016).

${ }^{37}$ I.-c. Yeh and G. Hummer, "System-size dependence of diffusion coefficients and viscosities from molecular dynamics simulations with periodic boundary conditions," J. Phys. Chem. B 108, 15873-15879 (2004).

${ }^{38}$ J. Irving and J. Kirkwood, "The statistical mechanics theory of transport processes. IV. The equations of hydrodynamics," J. Chem. Phys. 18, 817-829 (1950).
${ }^{39}$ P. Bordat and F. Müller-Plathe, "The shear viscosity of molecular fluids: A calculation by reverse nonequilibrium molecular dynamics," J. Chem. Phys. 116, 3362-3369 (2002).

${ }^{40}$ C. Niethammer, S. Becker, M. Bernreuther, M. Buchholz, W. Eckhardt, A. Heinecke, S. Werth, H.-J. Bungartz, C. W. Glass, H. Hasse, J. Vrabec, and M. Horsch, "Is1 mardyn: The massively parallel molecular dynamics code for large systems," J. Chem. Theory Comput. 10, 4455-4464 (2014).

${ }^{41} \mathrm{G}$. Rosenthal and S. H. L. Klapp, "Micelle and bilayer formation of amphiphilic Janus particles in a slit-pore," Int. J. Mol. Sci. 13, 9431-9446 (2012).

${ }^{42}$ J. G. Kirkwood and F. P. Buff, "The statistical mechanical theory of solutions. I," J. Chem. Phys. 19, 774-777 (1951).

${ }^{43}$ M. Heier, S. Stephan, J. Liu, W. G. Chapman, H. Hasse, and K. Langenbach, "Equation of state for the Lennard-Jones truncated and shifted fluid with a cutoff radius of $2.5 \sigma$ based on perturbation theory and its applications to interfacial thermodynamics," Mol. Phys. 116, 2083-2094 (2018).

${ }^{44}$ M. Thol, G. Rutkai, R. Span, J. Vrabec, and R. Lustig, "Equation of state for the Lennard-Jones truncated and shifted model fluid," Int. J. Thermophys. 36, 25-43 (2015).

${ }^{45}$ F. Ree, T. Ree, and H. Eyring, "Relaxation theory of transport problems in condensed systems," Ind. Eng. Chem. 50, 1036-1040 (1958).

${ }^{46}$ J. C. M. Li and P. Chang, "Self-diffusion coefficient and viscosity in liquids," J. Chem. Phys. 23, 518-520 (1955).

${ }^{47}$ D. J. Evans and H. Hanley, "A thermodynamics of steady homogeneous shear flow," Phys. Lett. A 80, 175-177 (1980).

${ }^{48}$ J. Ge, B. D. Todd, G. Wu, and R. J. Sadus, "Scaling behavior for the pressure and energy of shearing fluids," Phys. Rev. E: Stat. Phys., Plasmas, Fluids, Relat. Interdiscip. Top. 67, 061201 (2003).

${ }^{49} \mathrm{~K}$. Kawasaki and J. D. Gunton, "Theory of nonlinear transport processes: Nonlinear shear viscosity and normal stress effects," Phys. Rev. A 8, 2048-2064 (1973).

${ }^{50} \mathrm{M}$. Schmidt and H. Lipson, Eureqa (Version 0.98 Beta), Nutonian, Inc., Boston, 2014.

${ }^{51}$ G. Marcelli, B. D. Todd, and R. J. Sadus, "Analytic dependence of the pressure and energy of an atomic fluid under shear," Phys. Rev. E: Stat. Phys., Plasmas, Fluids, Relat. Interdiscip. Top. 63, 021204 (2001).

${ }^{52} \mathrm{~V}$. Garzó and M. López de Haro, "Tracer diffusion under shear flow for general repulsive interactions," Phys. Fluids 7, 478-486 (1995).

${ }^{53}$ V. Garzó and A. Santos, "Diffusion in a gaseous dilute solution under heat and momentum transport," Phys. Rev. E 52, 4942-4951 (1995).

${ }^{54} \mathrm{~S}$. Peter, "Über die viskosität und die selbstdiffusion von flüssigkeiten," $\mathrm{Z}$. Naturforsch. A 9, 98-104 (1954).

${ }^{55}$ A. Baranyai and P. T. Cummings, "Directional dependence of the random kinetic energy in planar Couette flow," Mol. Phys. 90, 35-41 (1997).

${ }^{56} \mathrm{H}$. Eyring, "Viscosity, plasticity, and diffusion as examples of absolute reaction rates," J. Chem. Phys. 4, 283-291 (1936).

${ }^{57}$ A. E. Giannakopoulos, F. Sofos, T. E. Karakasidis, and A. Liakopoulos, “Unified description of size effects of transport properties of liquids flowing in nanochannels," Int. J. Heat Mass Transfer 55, 5087-5092 (2012). 